\title{
Small-Signal Modeling and Analysis of Grid-Connected Inverter with Power Differential Droop Control
}

\author{
Xin Chen, Changhua Zhang, Qi Huang, and Mark Ofori-Oduro \\ School of Energy Science and Engineering, University of Electronic Science and Technology of China, Chengdu 611731, China \\ Correspondence should be addressed to Xin Chen; ainks517@163.com
}

Received 2 February 2016; Revised 1 June 2016; Accepted 2 June 2016

Academic Editor: Stefan Balint

Copyright (c) 2016 Xin Chen et al. This is an open access article distributed under the Creative Commons Attribution License, which permits unrestricted use, distribution, and reproduction in any medium, provided the original work is properly cited.

\begin{abstract}
The conventional voltage and frequency droop control strategy in grid-connected inverter suffers a major setback in the presence of disturbance by producing oscillations. Adding a power differential term in droop controller is an effective way to address such drawback. In this paper, grid-connected inverter's small-signal models of the conventional droop control and the power differential droop control are established. The eigenvalues of the models are then determined by system matrix. The eigenvalues analysis is presented which helps in identifying the relationship between the system stability and controller parameters. It is concluded that the damping ratio of dominant low-frequency eigenvalues increased and the oscillation caused by the disturbance is suppressed when a power differential term is added to the droop control method. The MATLAB/Simulink models of grid-connected inverter with both control strategies are also established to validate the results of small-signal analysis.
\end{abstract}

\section{Introduction}

The concept of distributed power generation and distributed energy storage has come to gain more attention because of the several advantages it offers over centralized power generation and storage [1-4]. Power electronic converters, typically the inverters, are used to interface these distributed generators (DGs) to the grid $[5,6]$. They make the sources more flexible to control and operate. However, their physical negligible inertia also makes the system prone to oscillations when subjected to disturbances.

DGs in a microgrid are expected to operate in an autonomous manner. And their operation and dynamics are strongly contingent upon the connected sources and on the power regulation control of the power electronic converters. The conventional means of control in DG is carried out by mimicking conventional generators. Based on this, the droop control is presented in [7-11]. A droop control scheme uses local power to detect changes in the system and adjust the operating points of generators accordingly. The scheme achieves accurate power sharing while maintaining regulation of the DG's voltage magnitude and frequency. However, the stability and performance of a control scheme are contingent on system parameters, their state, and the droop gain. Analysis of previous works has shown that the selection of the droop gain is crucial for the stability and performance of the DG. Empirical formulas are used in the selection of these gains [12]. However, it is not sufficient to analyze the consequences of the droop gains on the stability of the DG and microgrid as a whole. To address this problem, small-signal modeling is used [11-15].

On the other hand, the droop controller cannot provide sufficient low-frequency damping to compensate for the lack of the physical inertia in DG $[14,15]$. It still makes the system potentially susceptible to oscillation by disturbances. In conventional synchronous generators, power system stabilizer (PSS) is used to rectify the same oscillation problems in the output power. The PSS improves the damping of the electromechanical oscillations by acting through the generator excitation system to produce electrical torque in addition to the damping torque according to the speed deviation generated [16]. Using the same idea, it can be stipulated that adding a power differential term to the droop control is a possible means of solving the oscillation problem associated with the inverter in DGs. And it has been found to be effective in [15]. However, the stability and performance of the DG have not yet been fully analyzed and compared with 


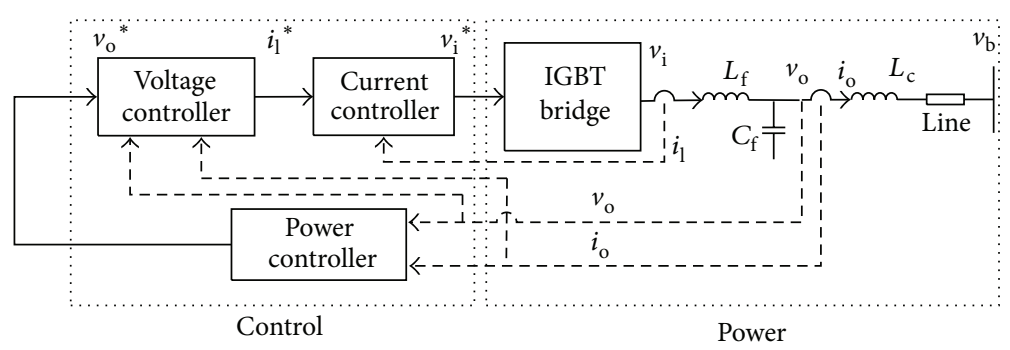

FIGURE 1: The circuit of inverter and the controller.

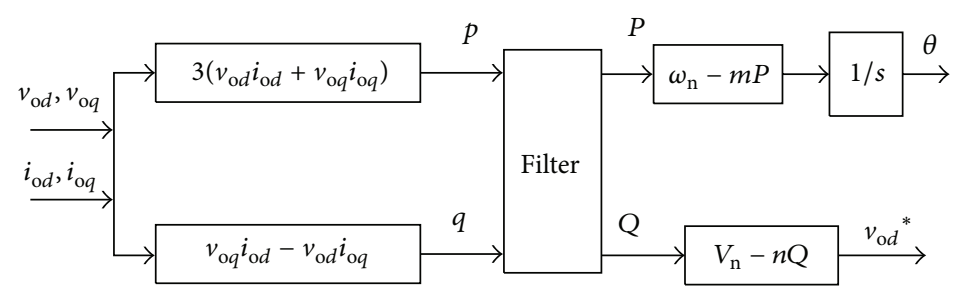

Figure 2: Power controller.

the droop control after the addition of the power differential term.

In this paper, complete small-signal models for DG are established for both the droop control and the power differential droop control. Further, the eigenvalue distribution of each control strategy is plotted to compare the damping ratios. Stability analysis of the DG under both control strategies is conducted in order to know the relation between stability and control parameters. Finally, MATLAB/Simulink simulations are used to validate the small-signal model and analysis.

The rest of the paper is in the following order. In Section 2, the structure of DG is introduced. In Section 3, a complete small-signal model for DG with the droop control is presented. Based on this work, in Section 4, modeling of DG with power differential droop control is presented. Stability analysis and simulation results are provided in Section 5. And conclusions are drawn in Section 6.

\section{Grid-Connected Inverter}

DG commonly interfaces with microgrid through voltage source inverter (VSI). As shown in Figure 1, VSI is interfaced with the microgrid at bus $v_{b}$. Modeling of the system is in two main sections: the VSI and the bus. The VSI is divided into two main parts: the power block and the control block. The three-phase inverter bridge, output LCL filter, and transmission line make up the power block, while the control block consists of the power controller, voltage controller, and current controller. The switching effect of the inverter can be ignored for cases of high frequency. Assuming an ideal voltage source for the inverter, the DC bus dynamics are also ignored. With the exception of the ignored components mentioned, state models are presented for all the above components in the next section.

\section{Model with Droop Control}

3.1. Power Controller. Figure 2 shows the block diagram of power controller, where $v_{\mathrm{o} d}, v_{\mathrm{o} q}, i_{\mathrm{o} d}$, and $i_{\mathrm{o} q}$ are $d$-q-axis component of inverter output voltage $v_{\mathrm{o}}$ and current $i_{\mathrm{o}}$. Here, $p$ is calculated as three-phase active power and $q$ as singlephase reactive power. These power components are passed through low-pass filters, shown as (1), to obtain the active power $P$ and reactive power $Q$. $\omega_{c}$ represents the cut-off frequency of low-pass filters. Hence,

$$
\begin{aligned}
& P=\frac{\omega_{c}}{s+\omega_{c}} p, \\
& Q=\frac{\omega_{c}}{s+\omega_{c}} q .
\end{aligned}
$$

To mimic the inertia characteristics of conventional generators and provide a degree of negative feedback, an artificial droop characteristic is introduced in the inverter frequency $\omega$ as in (2). $\omega$ is set according to the droop gains $m$ and $P$. And phase $\theta$ is set by integrating the frequency as in (3), where $\omega_{\mathrm{n}}$ is rated frequency. $P_{\text {rate }}$ is the rated active power. Hence,

$$
\begin{aligned}
& \omega=\omega_{\mathrm{n}}-m\left(P-P_{\text {rate }}\right), \\
& \theta=\omega_{\mathrm{n}} t-\int m\left(P-P_{\text {rate }}\right) d t .
\end{aligned}
$$

Similarly, a droop characteristic is also introduced in the voltage magnitude as given in (4). Here, the voltages are under the $d-q$ synchronous rotating frame with reference to the inverter's frequency. Therefore, the output voltage magnitude reference is represented at the $d$-axis, and the $q$-axis reference is set to zero. Therefore,

$$
\begin{aligned}
& v_{\mathrm{od}}{ }^{*}=V_{\mathrm{n}}-n \mathrm{Q}, \\
& {v_{\mathrm{o} q}}^{*}=0 .
\end{aligned}
$$




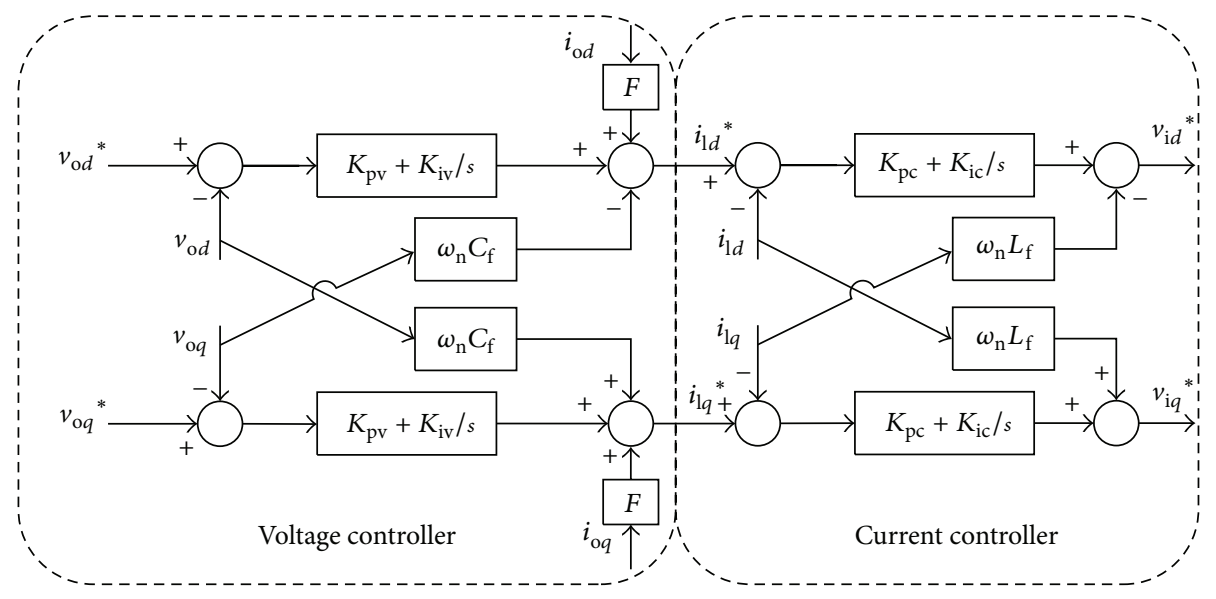

FIgURE 3: Voltage and current controller.

Equations (1) to (4) are then rearranged and linearized to obtain the state-space form as shown in

$$
\begin{aligned}
{\left[\begin{array}{c}
\Delta \delta_{1} \\
\Delta P \\
\Delta Q
\end{array}\right]=} & \mathbf{A}_{\mathbf{P}}\left[\begin{array}{c}
\Delta \delta_{1} \\
\Delta P \\
\Delta Q
\end{array}\right]+\mathbf{B}_{\mathbf{P}}\left[\begin{array}{c}
\Delta i_{1 d q} \\
\Delta v_{\text {odq }} \\
\Delta i_{\text {odq }}
\end{array}\right] \\
& +\mathbf{B}_{\mathbf{P} \omega c \mathrm{om}}\left[\Delta \omega_{\text {com }}\right], \\
{\left[\begin{array}{c}
\Delta \omega \\
\Delta v_{\mathrm{odq}}
\end{array}\right]=} & {\left[\begin{array}{c}
\mathbf{C}_{\mathbf{P} \boldsymbol{\omega}} \\
\mathbf{C}_{\mathbf{P v}}
\end{array}\right]\left[\begin{array}{c}
\Delta \delta \\
\Delta P \\
\Delta Q
\end{array}\right]+\left[\begin{array}{c}
\mathbf{D}_{\mathbf{P} \boldsymbol{\omega}} \\
\mathbf{D}_{\mathbf{P}_{\mathbf{v}}}
\end{array}\right]\left[\begin{array}{c}
\Delta i_{\text {ldq }} \\
\Delta v_{\text {odq }} \\
\Delta i_{\text {odq }}
\end{array}\right] . }
\end{aligned}
$$

All matrices in (5) have been shown in the Appendix, the same as below.

3.2. Voltage and Current Controller. The controllers for both the voltage and the current are shown in Figure 3. Both controllers employ standard proportional-integral (PI) regulators with decoupling and feedforward control loops. The voltage controller generates the reference vector for the current controller. Set outputs of integrator of PI regulators are $\varphi_{d}, \varphi_{q}$ (voltage controller) and $\gamma_{d}, \gamma_{q}$ (current controller). Then, the dynamics of the voltage and current controller can be given as

$$
\begin{aligned}
& i_{\mathrm{ld}}{ }^{*}=F i_{\mathrm{o} d}-\omega_{\mathrm{n}} C_{\mathrm{f}} v_{\mathrm{o} q}+K_{\mathrm{pv}}\left(v_{\mathrm{o} d}{ }^{*}-v_{\mathrm{o} d}\right)+K_{\mathrm{iv}} \varphi_{d}, \\
& i_{\mathrm{lq}}{ }^{*}=F i_{\mathrm{oq}}+\omega_{\mathrm{n}} C_{\mathrm{f}} v_{\mathrm{o} d}+K_{\mathrm{pv}}\left(v_{\mathrm{o} q}{ }^{*}-v_{\mathrm{o} q}\right)+K_{\mathrm{iv}} \varphi_{q}, \\
& v_{\mathrm{i} d}{ }^{*}=-\omega_{\mathrm{n}} L_{\mathrm{f}} i_{\mathrm{l} q}+K_{\mathrm{pc}}\left(i_{\mathrm{l} d}{ }^{*}-i_{\mathrm{l} d}\right)+K_{\mathrm{ic}} \gamma_{d}, \\
& {v_{\mathrm{i} q}}^{*}=\omega_{\mathrm{n}} L_{\mathrm{f}} i_{1 d}+K_{\mathrm{pc}}\left(i_{\mathrm{lq}}{ }^{*}-i_{\mathrm{lq}}\right)+K_{\mathrm{ic}} \gamma_{q},
\end{aligned}
$$

where $K_{\mathrm{pv}}$ and $K_{\mathrm{iv}}$ are the proportional and integral gains of voltage controller. $F$ is the feedforward gain. $K_{\mathrm{pc}}$ and $K_{\mathrm{ic}}$ are the proportional and integral gains of current controller.
The aforesaid equations can be linearized and the following state-space model of voltage and current controller unit can be obtained:

$$
\begin{aligned}
& {\left[\Delta \varphi_{d q}\right]=\mathbf{B}_{\mathrm{V} 1}\left[\Delta v_{\mathrm{o} d q}{ }^{*}\right]+\mathbf{B}_{\mathrm{V} 2}\left[\begin{array}{c}
\Delta i_{\mathrm{ldq}} \\
\Delta v_{\mathrm{odq}} \\
\Delta i_{\mathrm{odq}}
\end{array}\right],} \\
& {\left[\Delta i_{1 d q}{ }^{*}\right]=\mathbf{C}_{\mathbf{V}}\left[\Delta \varphi_{d q}\right]+\mathbf{D}_{\mathbf{V} \mathbf{1}}\left[\Delta v_{\mathrm{odq}}{ }^{*}\right]} \\
& +\mathbf{D}_{\mathbf{V} 2}\left[\begin{array}{lll}
\Delta i_{1 d q} & \Delta v_{\mathrm{odq}} & \Delta i_{\mathrm{odq}}
\end{array}\right]^{T}, \\
& {\left[\Delta \gamma_{d q}\right]=\mathbf{B}_{\mathbf{C} 1}\left[\Delta i_{1 d q}{ }^{*}\right]+\mathbf{B}_{\mathrm{C} 2}\left[\begin{array}{c}
\Delta i_{1 d q} \\
\Delta v_{\mathrm{odq}} \\
\Delta i_{\mathrm{odq}}
\end{array}\right],} \\
& {\left[\Delta v_{\mathrm{i} d q}{ }^{*}\right]=\mathbf{C}_{\mathbf{C}}\left[\Delta \gamma_{d q}\right]+\mathbf{D}_{\mathbf{C} 1}\left[\Delta i_{1 d q}{ }^{*}\right]} \\
& +\mathbf{D}_{\mathrm{C} 2}\left[\begin{array}{c}
\Delta i_{\mathrm{ldq}} \\
\Delta v_{\mathrm{odq}} \\
\Delta i_{\mathrm{odq}}
\end{array}\right] \text {. }
\end{aligned}
$$

3.3. Output LCL Filter and Line. Assuming that the inverter bridge losses are negligible, then $v_{\mathrm{i}}=v_{\mathrm{i}}{ }^{*}$. The output filter and line model can be represented by the following equations:

$$
\begin{aligned}
& \frac{d i_{1 d}}{d t}=\frac{-r_{\mathrm{f}}}{L_{\mathrm{f}}} i_{\mathrm{l} d}+\omega i_{\mathrm{lq}}+\frac{1}{L_{\mathrm{f}}} v_{\mathrm{i} d}-\frac{1}{L_{\mathrm{f}}} v_{\mathrm{od}}, \\
& \frac{d i_{\mathrm{lq}}}{d t}=\frac{-r_{\mathrm{f}}}{L_{\mathrm{f}}} i_{\mathrm{lq}}-\omega i_{\mathrm{ld}}+\frac{1}{L_{\mathrm{f}}} v_{\mathrm{i} q}-\frac{1}{L_{\mathrm{f}}} v_{\mathrm{o} q}, \\
& \frac{d v_{\mathrm{o} d}}{d t}=\omega v_{\mathrm{o} q}+\frac{1}{C_{\mathrm{f}}} i_{l d}-\frac{1}{C_{\mathrm{f}}} i_{\mathrm{o} d}, \\
& \frac{d v_{\mathrm{oq}}}{d t}=-\omega v_{\mathrm{o} d}+\frac{1}{C_{\mathrm{f}}} i_{\mathrm{lq}}-\frac{1}{C_{\mathrm{f}}} i_{\mathrm{oq}},
\end{aligned}
$$




$$
\begin{aligned}
& \frac{d i_{\mathrm{o} d}}{d t}=\frac{-\left(r_{\mathrm{c}}+r_{\mathrm{g}}\right)}{L_{\mathrm{c}}+L_{\mathrm{g}}} i_{\mathrm{o} d}+\omega i_{\mathrm{o} q}+\frac{1}{L_{\mathrm{c}}+L_{\mathrm{g}}}\left(v_{\mathrm{o} d}-v_{\mathrm{b} d}\right), \\
& \frac{d i_{\mathrm{o} q}}{d t}=\frac{-\left(r_{\mathrm{c}}+r_{\mathrm{g}}\right)}{L_{\mathrm{c}}+L_{\mathrm{g}}} i_{\mathrm{o} q}-\omega i_{\mathrm{o} d}+\frac{1}{L_{\mathrm{c}}+L_{\mathrm{g}}}\left(v_{\mathrm{o} q}-v_{\mathrm{b} q}\right),
\end{aligned}
$$

where $r_{\mathrm{f}}$ and $r_{\mathrm{c}}$ are equivalent resistances of $L_{\mathrm{f}}$ and $L_{\mathrm{c}}$. And $r_{\mathrm{g}}$ and $L_{\mathrm{g}}$ are equivalent resistance and inductance of line.

It can be seen that bus voltage $v_{\mathrm{b}}$ is the input of this model, and there is a phase difference $\left(\delta_{2}\right)$ between $v_{\mathrm{b}}$ and inverter's output voltage $v_{\mathrm{o}}$. Hence, $v_{\mathrm{b}}$ needs to be converted to the inverter's reference frame. The equations for transformation are shown as

$$
\begin{aligned}
& v_{\mathrm{bd}}=V_{\mathrm{bus}} \cos \delta_{2}, \\
& v_{\mathrm{b} q}=V_{\text {bus }} \sin \delta_{2},
\end{aligned}
$$

where $V_{\text {bus }}$ is the amplitude of $v_{\mathrm{b}}$.

Linearizing the above equations, the state-space model of the output filter and line is given by

$$
\begin{array}{r}
{\left[\begin{array}{c}
\Delta i_{\mathrm{ldq}} \\
\Delta v_{\mathrm{o} d q} \\
\Delta i_{\mathrm{o} d q}
\end{array}\right]=} \\
\mathbf{A}_{\mathrm{LCL}}\left[\begin{array}{c}
\Delta i_{\mathrm{ldq}} \\
\Delta v_{\mathrm{o} d q} \\
\Delta i_{\mathrm{o} d q}
\end{array}\right]+\mathbf{B}_{\mathrm{LCL} 1}\left[\Delta v_{\mathrm{i} d q}\right] \\
+\mathbf{B}_{\mathrm{LCL} 2}\left[\Delta \delta_{2}\right]+\mathbf{B}_{\mathrm{LCL} 3}[\Delta \omega] .
\end{array}
$$

3.4. Bus Model. Assume that the amplitude and frequency of the bus are constant. Then, $\delta_{2}$ changes according to the inverter's frequency. For simplicity, the inverter's reference frame is set as common reference frame. The dynamics of $\delta_{2}$ can then be given as

$$
\dot{\delta}_{2}=\omega_{\mathrm{n}}-\left(\omega_{\mathrm{n}}-m\left(P-P_{\text {rate }}\right)\right)=m\left(P-P_{\text {rate }}\right) .
$$

And the state-space model of the bus can be obtained as

$$
\left[\begin{array}{l}
\Delta \delta_{2}
\end{array}\right]=\mathbf{B}_{\text {bus }}\left[\begin{array}{lll}
\Delta \delta_{1} & \Delta P & \Delta Q
\end{array}\right]^{T} .
$$

3.5. Complete Model of the Inverter. A complete model of the grid-connected inverter is achieved by combining all submodels (5), (7), (10), and (12), and shown in

$$
\left[\Delta x_{\text {sys }}\right]=\mathbf{A}_{\text {sys }}\left[\Delta x_{\text {sys }}\right] \text {. }
$$

\section{Model with Power Differential Droop Control}

To improve inverter damping of low-frequency oscillation modes, by reference to PSS's operational principle, the droop characteristics are modified as follows [15]:

$$
\begin{aligned}
\omega & =\omega_{\mathrm{n}}-m\left(P-P_{\text {rate }}\right)-m_{\mathrm{d}} \frac{d P}{d t}, \\
v_{\mathrm{o} d}{ }^{*} & =V_{\mathrm{n}}-n Q-n_{\mathrm{d}} \frac{d Q}{d t} .
\end{aligned}
$$

TABLE 1: System parameters.

\begin{tabular}{lc}
\hline Parameter & Value \\
\hline$L_{\mathrm{f}}(\mathrm{mH})$ & 1.4 \\
$L_{\mathrm{c}}(\mathrm{mH})$ & 0.35 \\
$C_{\mathrm{f}}(\mu \mathrm{f})$ & 50 \\
$L_{\mathrm{g}}(\mu \mathrm{H})$ & 26.9 \\
$K_{\mathrm{pv}}$ & 0.05 \\
$K_{\mathrm{pc}}$ & 10.5 \\
$F$ & 0.75 \\
$V_{\mathrm{n}}(\mathrm{V})$ & 220 \\
$r_{\mathrm{f}}(\Omega)$ & 0.1 \\
$r_{\mathrm{c}}(\Omega)$ & 0.03 \\
$\omega_{\mathrm{c}}(\mathrm{rad} / \mathrm{s})$ & 31.41 \\
$r_{\mathrm{g}}(\Omega)$ & 0.22 \\
$K_{\mathrm{iv}}$ & 390 \\
$K_{\mathrm{ic}}$ & $16 \mathrm{e} 3$ \\
$\omega_{\mathrm{n}}(\mathrm{rad} / \mathrm{s})$ & 314.1 \\
$P_{\mathrm{rate}}(\mathrm{kW})$ & 10 \\
\hline
\end{tabular}

Since modification only affects the power controller (5) and bus (12) models, the previous power controller models are updated as in (15) so as to construct a new system model with the above modification. Hence,

$$
\begin{aligned}
& {\left[\begin{array}{c}
\Delta \delta_{1} \\
\Delta P \\
\Delta Q
\end{array}\right]=\mathbf{A}_{\mathbf{P} \_d t}\left[\begin{array}{c}
\Delta \delta_{1} \\
\Delta P \\
\Delta Q
\end{array}\right]+\mathbf{B}_{\mathbf{P} \_d t}\left[\begin{array}{c}
\Delta i_{1 d q} \\
\Delta v_{\text {odq }} \\
\Delta i_{\text {odq }}
\end{array}\right]} \\
& +\mathbf{B}_{\mathbf{P} \omega \text { com_dt }}\left[\Delta \omega_{\text {com }}\right] \text {, } \\
& {\left[\begin{array}{c}
\Delta \omega \\
\Delta v_{\mathrm{odq}}{ }^{*}
\end{array}\right]=\left[\begin{array}{c}
\mathbf{C}_{\mathbf{P} \boldsymbol{\omega} \_\mathrm{dt}} \\
\mathbf{C}_{\mathbf{P v} \_\mathrm{dt}}
\end{array}\right]\left[\begin{array}{c}
\Delta \delta \\
\Delta P \\
\Delta Q
\end{array}\right]+\left[\begin{array}{l}
\mathbf{D}_{\mathbf{P} \boldsymbol{\omega} \_\mathrm{dt}} \\
\mathbf{D}_{\mathbf{P v \_ d t}}
\end{array}\right]\left[\begin{array}{c}
\Delta i_{\mathrm{ldq}} \\
\Delta v_{\mathrm{o} d q} \\
\Delta i_{\mathrm{odq}}
\end{array}\right] .}
\end{aligned}
$$

The bus model with power differential term is modified as follows:

$$
\left[\begin{array}{r}
\Delta \delta_{2}
\end{array}\right]=\mathbf{B}_{\text {bus } 1}\left[\begin{array}{lll}
\Delta \delta_{1} & \Delta P & \Delta Q
\end{array}\right]^{T}+\mathbf{B}_{\text {bus } 2}\left[\begin{array}{c}
\Delta i_{1 d q} \\
\Delta v_{\mathrm{o} d q} \\
\Delta i_{\mathrm{o} d q}
\end{array}\right] .
$$

The system model with power differential term droop control is represented as

$$
\left[\Delta \dot{x}_{\text {sys }}\right]=\mathbf{A}_{\text {sys_dt }}\left[\Delta x_{\text {sys }}\right] \text {. }
$$

\section{Stability Analysis and Simulation}

To evaluate and compare the performances of both control schemes, according to Figure 1, simulation models have been built via MATLAB/Simulink. System parameters are given in Table 1, and initial conditions of the system are given in Table 2 . These steady-state operating point conditions were 
TABLE 2: Initial conditions.

\begin{tabular}{lc}
\hline State & Value \\
\hline$P(\mathrm{~kW})$ & 10 \\
$i_{1 d}(\mathrm{~A})$ & 15.13 \\
$v_{\mathrm{od}}(\mathrm{V})$ & 220.3 \\
$i_{\mathrm{od}}(\mathrm{A})$ & 15.13 \\
$\delta_{1}($ degree $)$ & 0 \\
$\omega(\mathrm{rad} / \mathrm{s})$ & 314.1 \\
$\mathrm{Q}(\mathrm{kW})$ & -18.5 \\
$i_{1 q}(\mathrm{~A})$ & 31.48 \\
$v_{\mathrm{oq}}(\mathrm{V})$ & 0 \\
$i_{\mathrm{oq}}(\mathrm{A})$ & 28.02 \\
$\delta_{2}($ degree $)$ & 2.3 \\
\hline
\end{tabular}

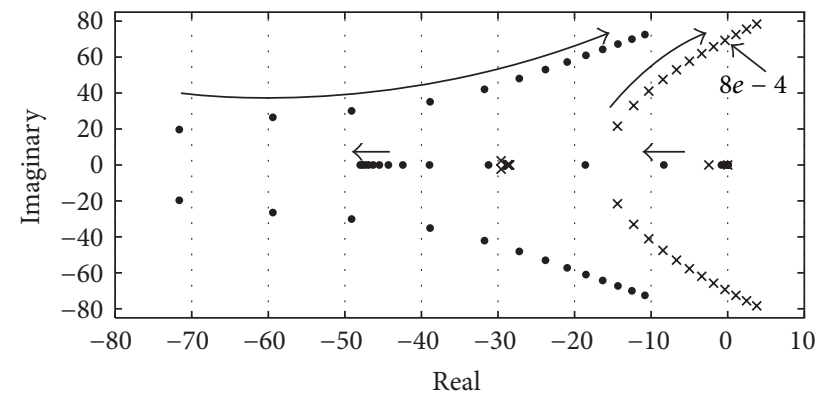

$\times$ Droop control

- Power differential droop control

FIGURE 4: Dominant locus of eigenvalue with $m$ increasing.

obtained from a MATLAB/Simulink time-step simulation of the system.

According to the established small-signal models, all eigenvalues calculations are based on Table 2 . The eigenvalues of high and intermediate frequency modes have weak influence on the stability of the system [14]. Therefore, in this paper, point of focus of analysis is on the low-frequency modes.

\subsection{Analysis for Droop Gains $m$ and $n$}

5.1.1. Active Power Droop Gain m. Figure 4 shows the dominant low-frequency eigenvalues as a function of the active power droop gain $m(1 e-5 \leq m \leq 1 e-3)$, the reactive power droop gain $n=5 e-5$, and the power differential gain $m_{\mathrm{d}}=n_{\mathrm{d}}=8 e-6$. It shows that as $m$ is increased, the complexconjugate pair of the dominant eigenvalues of the two control strategies move towards unstable region making the system more oscillatory. When $m=8 e-4$, the dominant eigenvalues of the droop control enter the right half plane, eventually leading to instability. Contrary to that, the eigenvalues of the power differential droop control stay on the left half plane. These trajectories show that, under the same condition, the range of $m$ increases when the power differential term is added to the conventional droop control. Figure 4 also shows that the complex-conjugate eigenvalues of the power differential droop control have a larger absolute real value than the droop control, which means that power differential term increases system damping and contributes to system stability.

In order to verify the correctness of the above smallsignal stability analysis, an effective method that selects some parameters which lead to system instability or oscillation is carried out. Then, observe whether the response of simulation model is consistent with the small-signal analysis.

Figure 5 shows output power response for 20\% step change in load, when $m=8 e-4$ for the two control strategies. It can be seen that, after the disturbance, the output power with the droop control starts to oscillate and eventually results in instability, while output power with the power differential droop control is steady.

Figure 6 shows the same 20\% step change in load when $m=4 e-4$ for the two control strategies. From the calculation result of eigenvalues, when $m=4 e-4$, the complex-conjugate eigenvalues of two control strategies are $-6.9 \pm 52.2 i$ (droop control) and $-27.7 \pm 47.4 i$ (power differential droop control) with damping ratios 0.13 (droop control) and 0.5 (power differential droop control). From Figure 6, it can be seen that the active power of the power differential droop control has less oscillation than the droop control. In Figure 6(a), it can be seen that the oscillation period is $0.12 \mathrm{~s}$, which is the same oscillation period given by complex-conjugate eigenvalues. These results validate the small-signal model and the analysis.

5.1.2. Reactive Power Gain n. Figure 7 shows the dominant low-frequency eigenvalues as a function of the reactive power droop gain $n(1 e-5 \leq n \leq 7.5 e-4)$, the active power droop gain $m=8 e-5$, and the power differential gain $m_{\mathrm{d}}=n_{\mathrm{d}}=8 e-6$. It can be seen that as $n$ is increased, the complex-conjugate dominant eigenvalues of the two control strategies move away from the imaginary axis, and the real eigenvalues move towards the right half plane, eventually leading the system to instability. It can also be seen that the power differential droop control has larger damping than the droop control at low-frequency oscillation modes.

Figure 8 shows the output power response for $20 \%$ step change in load when $n=5 e-4$ for the two control strategies. Again, from the calculation result of eigenvalues, when $n=$ $5 e-4$, the eigenvalues of the two control strategies are both in the left half plane, so both systems are stable. The complexconjugate eigenvalues of the droop control are $-25.4 \pm 24.7 i$; its given oscillation period of $0.25 \mathrm{~s}$ and damping ratio of 0.72 correspond to rapid decay of oscillation. The complexconjugate eigenvalues of the power differential droop control are $-124 \pm 54 i$, and the real eigenvalue is -16.7 , so the real eigenvalues play a dominant role; there is no low-frequency oscillation mode. It can be observed that the results in Figure 8 closely match the above analysis.

The simulation results in Figures 5, 6, and 8 are consistent with the stability and dynamic characteristics given by the small-signal model, demonstrating the validity of the smallsignal modeling and analysis. Considering the need for stability and suitable damping, this paper takes $m=8 e-5$ and $n=5 e-5$. 


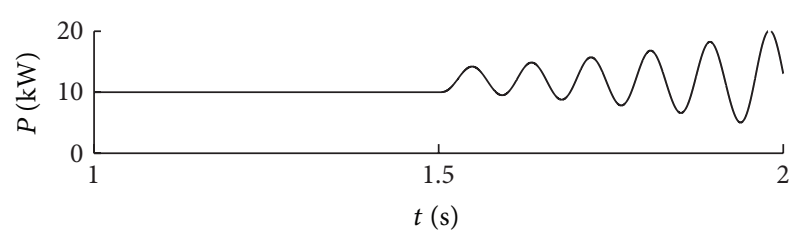

(a) Droop control

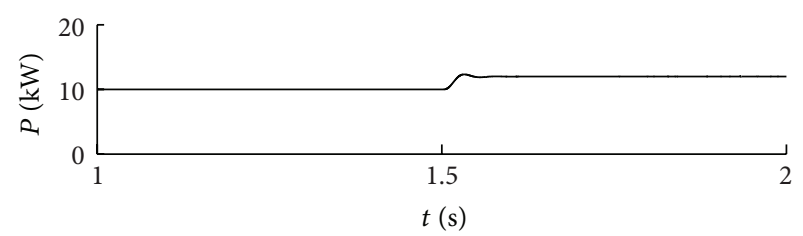

(b) Power differential droop control

FIGURE 5: Waveforms of active power when $m=8 e-4$.

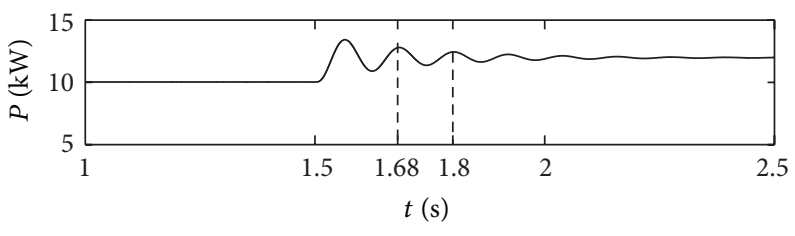

(a) Droop control

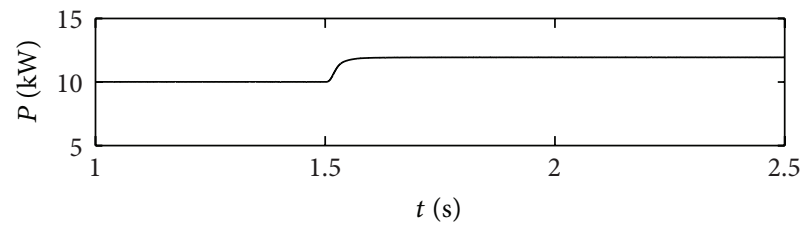

(b) Power differential droop control

FIGURE 6: Waveforms of active power when $m=4 e-4$.

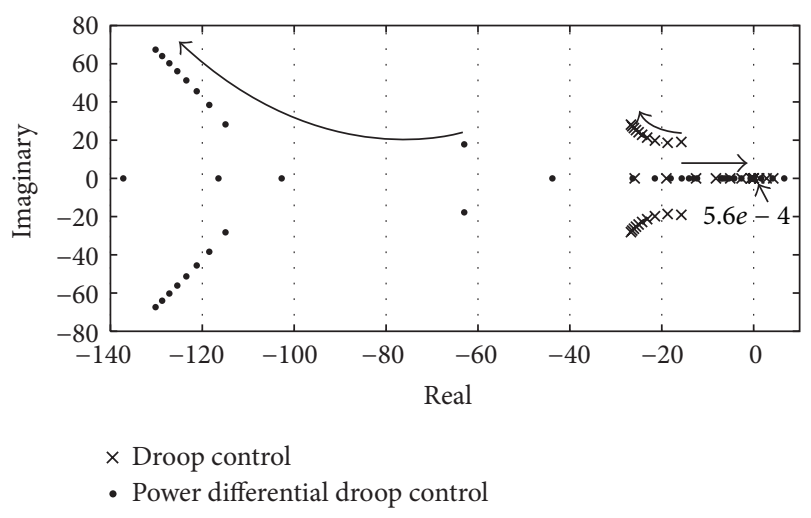

Figure 7: Dominant locus of eigenvalue with $n$ increasing.

5.2. Analysis for Power Differential Gains $m_{\mathrm{d}}, n_{\mathrm{d}}$. Figure 9 shows the dominant low-frequency eigenvalues as a function of the power differential gains $m_{\mathrm{d}}, n_{\mathrm{d}}\left(1 e-7 \leq m_{\mathrm{d}}, n_{\mathrm{d}} \leq 3 e-5\right)$. It can be seen that increasing $m_{\mathrm{d}}, n_{\mathrm{d}}$ causes the complexconjugate dominant eigenvalues of the power differential droop to move in a spiral manner. At the initial stage of the increment, the eigenvalues move away from the right half plane while approaching the real axis. However, further increment causes the eigenvalues to move away from the real axis and close to the right half plane, eventually moving into the right half plane. Therefore, selecting small values for $m_{\mathrm{d}}$, $n_{\mathrm{d}}$ causes the system to have small damping. Selecting large values also causes the system to have more oscillations in case of a disturbance, eventually leading to instability.

Figure 10 shows the output power response for the power differential droop control for a $20 \%$ step in load when $m_{\mathrm{d}}$, $n_{\mathrm{d}}$ take values of $1 e-7,4 e-6$, and $2.75 e-5$ under initial conditions. It can be seen that the output power in Figure 10(a) has more oscillations than that in Figure 10(b), which is because when $m_{\mathrm{d}}, n_{\mathrm{d}}=1 e-7$, the system damping is smaller than when $m_{\mathrm{d}}, n_{\mathrm{d}}=4 e-6$. In Figure $10(\mathrm{c})$, it can be seen that the system becomes unstable after the load step. This however is expected because the value of $m_{\mathrm{d}}, n_{\mathrm{d}}$ in this case moves the dominant complex-conjugate eigenvalues into the right half plane. Obviously, $4 e-6$ is an appropriate value of $m_{\mathrm{d}}$ and $n_{\mathrm{d}}$, when $m=8 e-5$ and $n=5 e-5$.

\section{Conclusion}

The grid-connected inverter with the droop control is potentially susceptible to oscillation due to its poor damping of lowfrequency mode. A solution is adding power differential term in droop controller. In this paper, the small-signal models of grid-connected inverter with both the droop control and the power differential droop control are established. The eigenvalues of these models were analyzed. And the following conclusions are obtained: the damping increased after adding power differential term, and the system oscillation is suppressed. The range of active power droop gain $m$ is increased compared with the droop control under stable conditions. The system with the power differential droop control has poor damping both on the small and on the large power differential gains $m_{\mathrm{d}}, n_{\mathrm{d}}$. And the system will be unstable with larger $m_{\mathrm{d}}, n_{\mathrm{d}}$. These results were verified by simulation model via MATLAB/Simulink. It was observed that the simulation system dynamics closely match the conclusion of analysis.

\section{Appendix}

\section{A. Matrices in Section 3}

A.1. Power Controller. One has the following:

$$
\left[\begin{array}{c}
\Delta i_{\mathrm{ldq}} \\
\Delta v_{\mathrm{odq}} \\
\Delta i_{\mathrm{odq}}
\end{array}\right]=\left[\begin{array}{llllll}
\Delta i_{\mathrm{ld}} & \Delta i_{\mathrm{lq}} & \Delta v_{\mathrm{od}} & \Delta v_{\mathrm{oq}} & \Delta i_{\mathrm{od}} & \Delta i_{\mathrm{oq}}
\end{array}\right]^{T},
$$




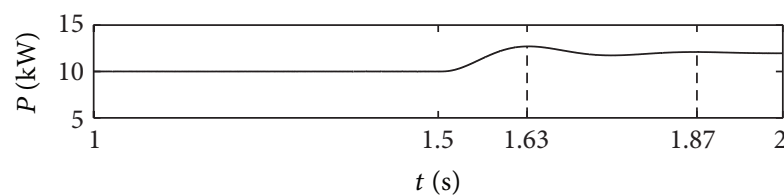

(a) Droop control

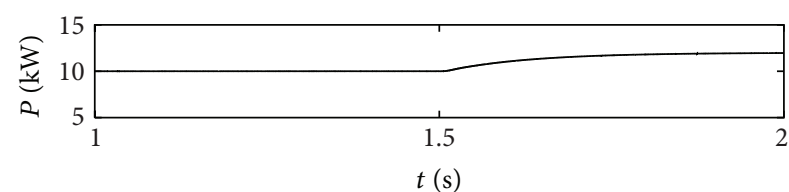

(b) Power differential droop control

FIGURE 8: Waveforms of active power when $n=5 e-4$.

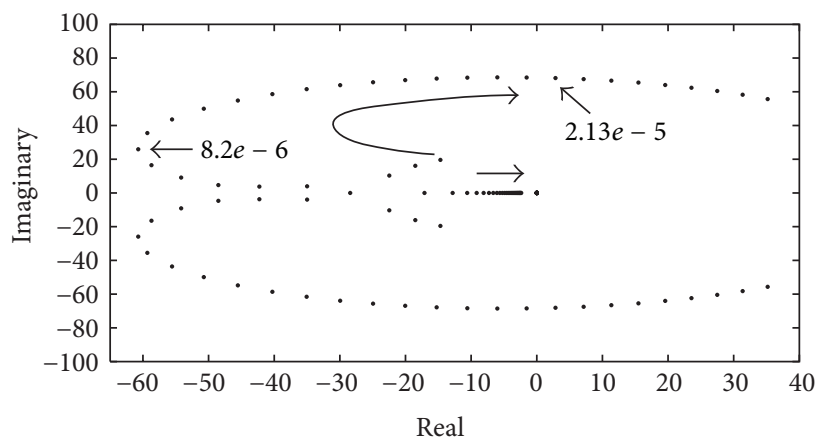

FIgURE 9: Dominant locus of eigenvalue with $m_{\mathrm{d}}, n_{\mathrm{d}}$ increasing.

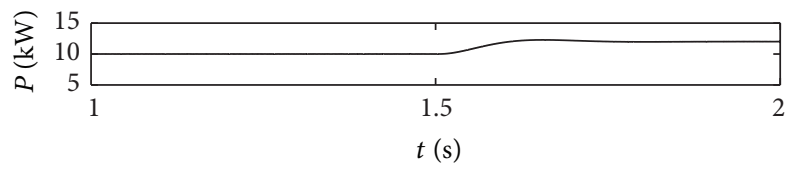

(a) $m_{\mathrm{d}}=n_{\mathrm{d}}=1 e-7$

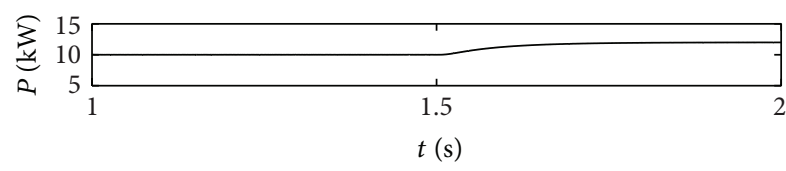

(b) $m_{\mathrm{d}}=n_{\mathrm{d}}=4 e-6$

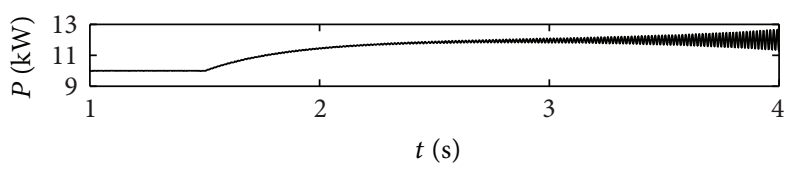

(c) $m_{\mathrm{d}}=n_{\mathrm{d}}=2.75 e-5$

FIGURE 10: Waveforms of active power in different $m_{\mathrm{d}}, n_{\mathrm{d}}$.

$$
\begin{aligned}
& \mathbf{A}_{\mathbf{P}}=\left[\begin{array}{ccc}
0 & -m & 0 \\
0 & -\omega_{c} & 0 \\
0 & 0 & -\omega_{c}
\end{array}\right] \\
& \mathbf{B}_{\mathbf{P} \omega \mathrm{com}}=\left[\begin{array}{c}
-1 \\
0 \\
0
\end{array}\right] \mathbf{D}_{\mathbf{P v}}=0_{2 \times 3} \\
& \mathbf{B}_{\mathbf{P}}=\left[\begin{array}{cccccc}
0 & 0 & 0 & 0 & 0 & 0 \\
0 & 0 & 3 \omega_{\mathrm{c}} I_{\mathrm{o} d} & 3 \omega_{\mathrm{c}} I_{\mathrm{oq}} & 3 \omega_{\mathrm{c}} V_{\mathrm{o} d} & 3 \omega_{\mathrm{c}} V_{\mathrm{oq}} \\
0 & 0 & \omega_{\mathrm{c}} I_{\mathrm{o} q} & -\omega_{\mathrm{c}} I_{\mathrm{o} d} & -\omega_{\mathrm{c}} V_{\mathrm{oq}} & \omega_{\mathrm{c}} V_{\mathrm{o} d}
\end{array}\right], \\
& \mathbf{C}_{\mathbf{P} \boldsymbol{\omega}}=\left[\begin{array}{lll}
0 & -m & 0
\end{array}\right], \\
& \mathbf{C}_{\mathbf{P v}}=\left[\begin{array}{ccc}
0 & 0 & -n \\
0 & 0 & 0
\end{array}\right] \mathbf{D}_{\mathbf{P} \boldsymbol{\omega}}=0_{1 \times 3} \text {. }
\end{aligned}
$$

\section{A.2. Voltage Controller. One has the following:}

$$
\begin{aligned}
& \Delta \varphi_{d q}=\left[\begin{array}{ll}
\Delta \varphi_{d} & \Delta \varphi_{q}
\end{array}\right]^{T}, \\
& \mathbf{B}_{\mathbf{V 1}}=\left[\begin{array}{ll}
1 & 0 \\
0 & 1
\end{array}\right] \text {, } \\
& \mathbf{B}_{\mathbf{V} 2}=\left[\begin{array}{cccccc}
0 & 0 & -1 & 0 & 0 & 0 \\
0 & 0 & 0 & -1 & 0 & 0
\end{array}\right] \text {, } \\
& \mathrm{C}_{\mathrm{V}}=\left[\begin{array}{cc}
K_{\mathrm{iv}} & 0 \\
0 & K_{\mathrm{iv}}
\end{array}\right] \text {, } \\
& \mathbf{D}_{\mathrm{V} \mathbf{1}}=\left[\begin{array}{cc}
K_{\mathrm{pv}} & 0 \\
0 & K_{\mathrm{pv}}
\end{array}\right] \text {, } \\
& \mathbf{D}_{\mathbf{V} 2}=\left[\begin{array}{cccccc}
0 & 0 & -K_{\mathrm{pv}} & -\omega_{\mathrm{n}} C_{\mathrm{f}} & F & 0 \\
0 & 0 & \omega_{\mathrm{n}} C_{\mathrm{f}} & -K_{\mathrm{pv}} & 0 & F
\end{array}\right] \text {. }
\end{aligned}
$$


And, for current controller,

$$
\begin{aligned}
& \Delta \gamma_{d q}=\left[\begin{array}{ll}
\Delta \gamma_{d} & \Delta \gamma_{q}
\end{array}\right]^{T}, \\
& \mathbf{B}_{\mathrm{C1}}=\left[\begin{array}{ll}
1 & 0 \\
0 & 1
\end{array}\right] \text {, } \\
& \mathbf{B}_{\mathbf{C} 2}=\left[\begin{array}{cccccc}
-1 & 0 & 0 & 0 & 0 & 0 \\
0 & -1 & 0 & 0 & 0 & 0
\end{array}\right], \\
& \mathbf{C}_{\mathbf{C}}=\left[\begin{array}{cc}
K_{\mathrm{ic}} & 0 \\
0 & K_{\mathrm{ic}}
\end{array}\right] \text {, } \\
& \mathbf{D}_{\mathrm{C} \mathbf{1}}=\left[\begin{array}{cc}
K_{\mathrm{pc}} & 0 \\
0 & K_{\mathrm{pc}}
\end{array}\right] \text {, } \\
& \mathbf{D}_{\mathbf{C} 2}=\left[\begin{array}{cccccc}
-K_{\mathrm{pc}} & -\omega_{\mathrm{n}} L_{\mathrm{f}} & 0 & 0 & 0 & 0 \\
\omega_{\mathrm{n}} L_{\mathrm{f}} & -K_{\mathrm{pc}} & 0 & 0 & 0 & 0
\end{array}\right] .
\end{aligned}
$$

A.3. Output LCL Filter and Line. One has the following:

$\mathbf{A}_{\text {LCL }}$

$$
=\left[\begin{array}{cccccc}
\frac{-r_{\mathrm{f}}}{L_{\mathrm{f}}} & \omega & \frac{-1}{L_{\mathrm{f}}} & 0 & 0 & 0 \\
-\omega & \frac{-r_{\mathrm{f}}}{L_{\mathrm{f}}} & 0 & \frac{-1}{L_{\mathrm{f}}} & 0 & 0 \\
\frac{1}{C_{\mathrm{f}}} & 0 & 0 & \omega & \frac{-1}{C_{\mathrm{f}}} & 0 \\
0 & \frac{1}{C_{\mathrm{f}}} & -\omega & 0 & 0 & \frac{-1}{C_{\mathrm{f}}} \\
0 & 0 & \frac{1}{L_{\mathrm{c}}+L_{\mathrm{g}}} & 0 & \frac{-\left(r_{\mathrm{c}}+r_{\mathrm{g}}\right)}{L_{\mathrm{c}}+L_{\mathrm{g}}} & \omega \\
0 & 0 & 0 & \frac{1}{L_{\mathrm{c}}+L_{\mathrm{g}}} & -\omega & \frac{-\left(r_{\mathrm{c}}+r_{\mathrm{g}}\right)}{L_{\mathrm{c}}+L_{\mathrm{g}}}
\end{array}\right],
$$

$$
\begin{aligned}
\mathbf{B}_{\mathrm{LCL1}} & =\left[\begin{array}{cc}
\frac{1}{L_{\mathrm{f}}} & 0 \\
0 & \frac{1}{L_{\mathrm{f}}} \\
0 & 0 \\
0 & 0 \\
0 & 0 \\
0 & 0
\end{array}\right], \\
\mathbf{B}_{\mathrm{LCL} 2} & =\left[\begin{array}{c}
0 \\
0 \\
0 \\
\frac{V_{\mathrm{bus}} \sin \delta_{2}}{L_{\mathrm{c}}+L_{\mathrm{g}}} \\
\frac{-V_{\mathrm{bus}} \cos \delta_{2}}{L_{\mathrm{c}}+L_{\mathrm{g}}}
\end{array}\right],
\end{aligned}
$$$$
\mathbf{B}_{\mathrm{LCL} 3}=\left[\begin{array}{llllll}
I_{\mathrm{lq}} & -I_{\mathrm{ld}} & V_{\mathrm{o} q} & -V_{\mathrm{o} d} & I_{\mathrm{o} q} & -I_{\mathrm{o} d}
\end{array}\right]^{T} .
$$

A.4. Bus Model. One has the following:

$$
\mathbf{B}_{\text {bus }}=\left[\begin{array}{lll}
0 & m & 0
\end{array}\right] \text {. }
$$

A.5. Complete Model of the Inverter. One has the following:

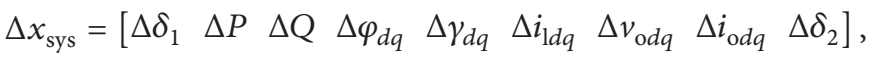

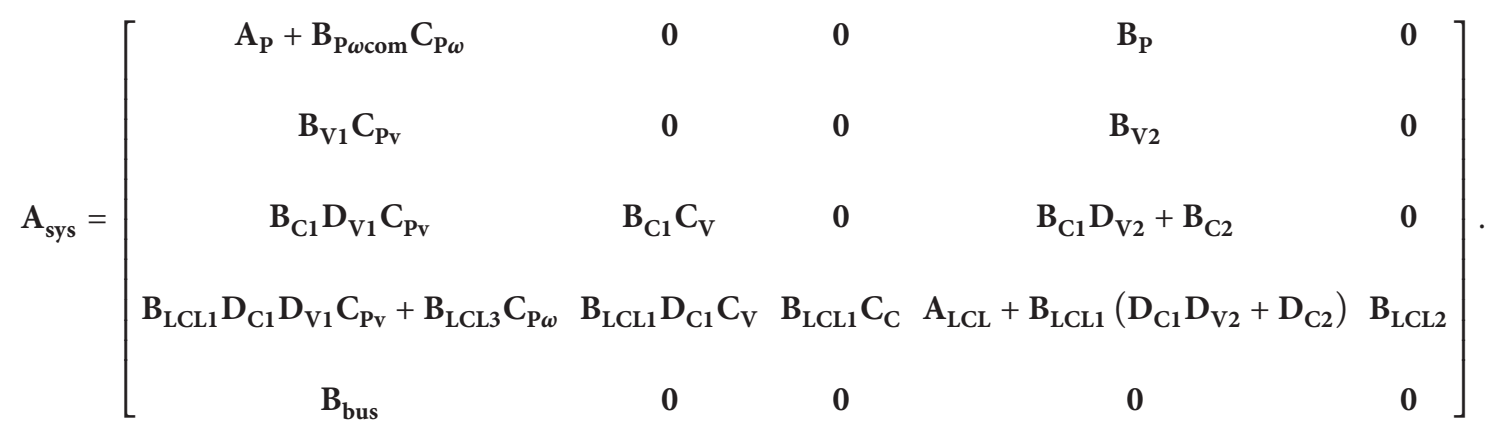




\section{B. Matrices in Section 4}

One has the following:

$$
\begin{aligned}
& \mathbf{A}_{\mathbf{P} \_d t}=\left[\begin{array}{ccc}
0 & -m+m_{\mathrm{d}} \omega_{\mathrm{c}} & 0 \\
0 & -\omega_{\mathrm{c}} & 0 \\
0 & 0 & -\omega_{\mathrm{c}}
\end{array}\right] \\
& \mathbf{B}_{\mathbf{P} \omega \text { com_dt }}=\left[\begin{array}{c}
-1 \\
0 \\
0
\end{array}\right] \\
& \mathbf{C}_{\mathbf{P} \omega_{-} \mathrm{dt}}=\left[\begin{array}{lll}
0 & -m & 0
\end{array}\right], \\
& \mathbf{C}_{\mathbf{P v} \_\mathrm{dt}}=\left[\begin{array}{ccc}
0 & 0 & -n \\
0 & 0 & 0
\end{array}\right] \text {, } \\
& \mathbf{B}_{\mathbf{P} \_d t}=\left[\begin{array}{cccccc}
0 & 0 & -3 m_{\mathrm{d}} \omega_{\mathrm{c}} I_{\mathrm{o} d} & -3 m_{\mathrm{d}} \omega_{\mathrm{c}} I_{\mathrm{o} q} & -3 m_{\mathrm{d}} \omega_{\mathrm{c}} V_{\mathrm{o} q} & -3 m_{\mathrm{d}} \omega_{\mathrm{c}} V_{\mathrm{o} d} \\
0 & 0 & 3 \omega_{\mathrm{c}} I_{\mathrm{o} d} & 3 \omega_{\mathrm{c}} I_{\mathrm{o} q} & 3 \omega_{\mathrm{c}} V_{\mathrm{o} d} & 3 \omega_{\mathrm{c}} V_{\mathrm{o} q} \\
0 & 0 & \omega_{\mathrm{c}} I_{\mathrm{o} q} & -\omega_{\mathrm{c}} I_{\mathrm{o} d} & -\omega_{\mathrm{c}} V_{\mathrm{o} q} & \omega_{\mathrm{c}} V_{\mathrm{o} d}
\end{array}\right] \text {, }
\end{aligned}
$$

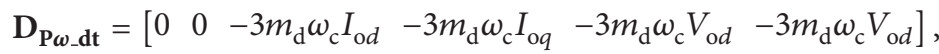

$$
\begin{aligned}
& \mathbf{D}_{\mathbf{P v} \_\mathbf{d t}}=\left[\begin{array}{cccccc}
0 & 0 & -n_{\mathrm{d}} \omega_{\mathrm{c}} I_{\mathrm{oq}} & n_{\mathrm{d}} \omega_{\mathrm{c}} I_{\mathrm{od}} & n_{\mathrm{d}} \omega_{\mathrm{c}} V_{\mathrm{oq}} & -n_{\mathrm{d}} \omega_{\mathrm{c}} V_{\mathrm{od}} \\
0 & 0 & 0 & 0 & 0 & 0
\end{array}\right] \text {, }
\end{aligned}
$$

$$
\begin{aligned}
& A_{\text {sys_dt }}
\end{aligned}
$$

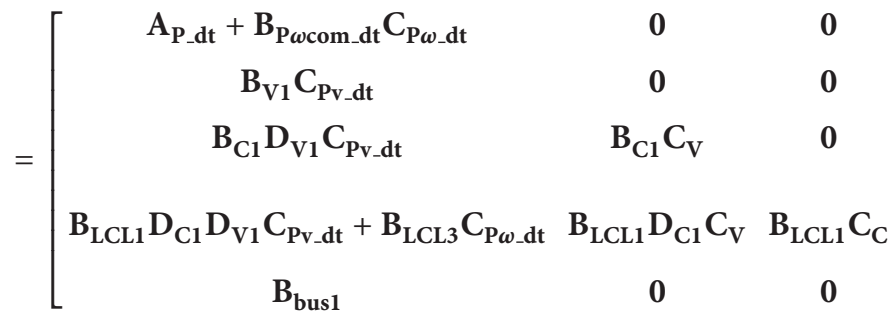

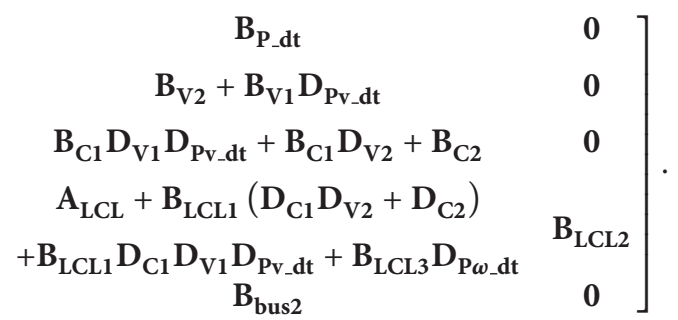

\section{Competing Interests}

The authors declare that they have no competing interests.

\section{References}

[1] F. Blaabjerg, R. Teodorescu, M. Liserre, and A. V. Timbus, "Overview of control and grid synchronization for distributed power generation systems," IEEE Transactions on Industrial Electronics, vol. 53, no. 5, pp. 1398-1409, 2006.

[2] V. C. Gungor, B. Lu, and G. P. Hancke, "Opportunities and challenges of wireless sensor networks in smart grid," IEEE Transactions on Industrial Electronics, vol. 57, no. 10, pp. 35573564, 2010.

[3] I. J. Balaguer, Q. Lei, S. Yang, U. Supatti, and F. Z. Peng, "Control for grid-connected and intentional islanding operations of distributed power generation," IEEE Transactions on Industrial Electronics, vol. 58, no. 1, pp. 147-157, 2011.
[4] T. L. Vandoorn, J. D. M. De Kooning, B. Meersman, J. M. Guerrero, and L. Vandevelde, "Voltage-Based control of a smart transformer in a microgrid," IEEE Transactions on Industrial Electronics, vol. 60, no. 4, pp. 1291-1305, 2013.

[5] J. He, Y. W. Li, D. Bosnjak, and B. Harris, "Investigation and active damping of multiple resonances in a parallel-inverterbased microgrid," IEEE Transactions on Power Electronics, vol. 28, no. 1, pp. 234-246, 2013.

[6] J. M. Guerrero, L. García de, M. Vicuna, and J. Miret, "Wireless control strategy for parallel operation of distributed-generation inverters," IEEE Transactions on Industrial Electronics, vol. 53, no. 5, pp. 1461-1470, 2006.

[7] D. De and V. Ramanarayanan, "Decentralized parallel operation of inverters sharing unbalanced and nonlinear loads," IEEE Transactions on Power Electronics, vol. 25, no. 12, pp. 3015-3025, 2010.

[8] M. C. Chandorkar, D. M. Divan, and R. Adapa, "Control of parallel connected inverters in standalone ac supply systems," 
IEEE Transactions on Industry Applications, vol. 29, no. 1, pp. 136-143, 1993.

[9] K. De Brabandere, B. Bolsens, J. Van den Keybus, A. Woyte, J. Driesen, and R. Belmans, "A voltage and frequency droop control method for parallel inverters," IEEE Transactions on Power Electronics, vol. 22, no. 4, pp. 1107-1115, 2007.

[10] H. Bevrani and S. Shokoohi, "An intelligent droop control for simultaneous voltage and frequency regulation in Islanded microgrids," IEEE Transactions on Smart Grid, vol. 4, no. 3, pp. 1505-1513, 2013.

[11] J. Kim, J. M. Guerrero, P. Rodriguez, R. Teodorescu, and K. Nam, "Mode adaptive droop control with virtual output impedances for an inverter-based flexible AC microgrid," IEEE Transactions on Power Electronics, vol. 26, no. 3, pp. 689-701, 2011.

[12] H. Liang, B. J. Choi, W. Zhuang, and X. Shen, "Stability enhancement of decentralized inverter control through wireless communications in microgrids," IEEE Transactions on Smart Grid, vol. 4, no. 1, pp. 321-331, 2013.

[13] A. K. Abbasi and M. W. Mustafa, "Mathematical model and stability analysis of inverter-based distributed generator," Mathematical Problems in Engineering, vol. 2013, Article ID 195038, 7 pages, 2013.

[14] N. Pogaku, M. Prodanović, and T. C. Green, "Modeling, analysis and testing of autonomous operation of an inverter-based microgrid," IEEE Transactions on Power Electronics, vol. 22, no. 2, pp. 613-625, 2007.

[15] Y. A.-R. I. Mohamed and E. F. El-Saadany, "Adaptive decentralized droop controller to preserve power sharing stability of paralleled inverters in distributed generation microgrids," IEEE Transactions on Power Electronics, vol. 23, no. 6, pp. 2806-2816, 2008.

[16] P. Kundur, Power System Stability and Control, McGraw-Hill, New York, NY, USA, 1994. 


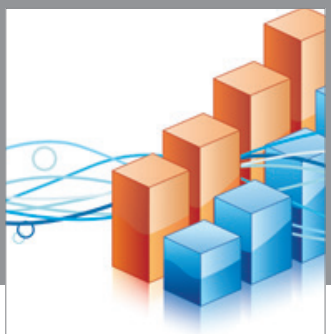

Advances in

Operations Research

vatem alat4

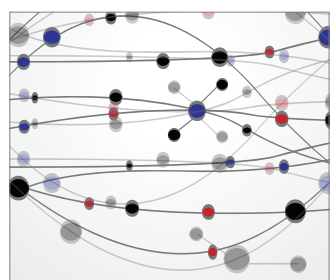

\section{The Scientific} World Journal
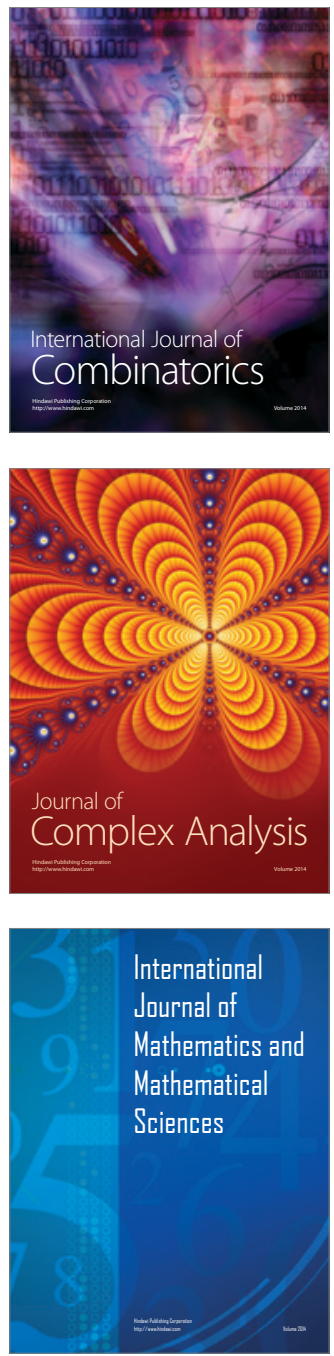
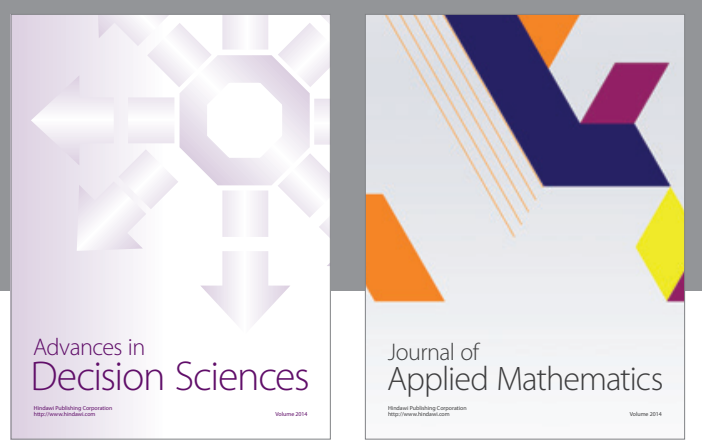

Algebra

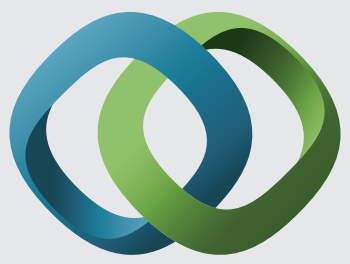

\section{Hindawi}

Submit your manuscripts at

http://www.hindawi.com
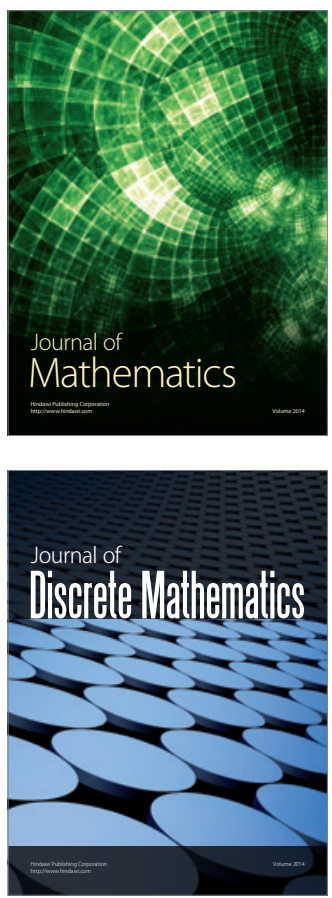

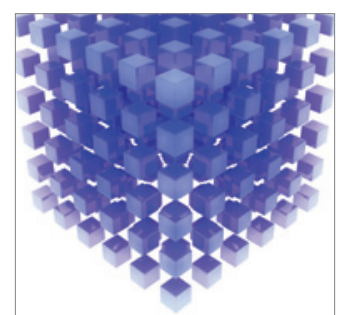

Mathematical Problems in Engineering
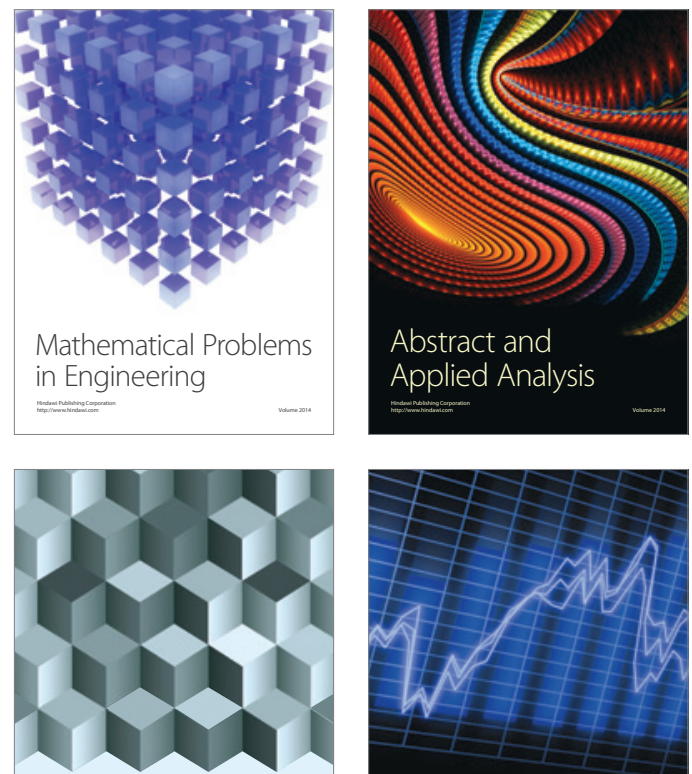

Journal of

Function Spaces

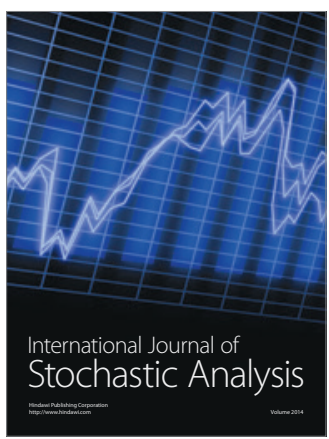

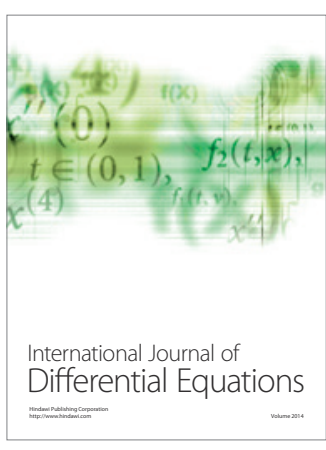
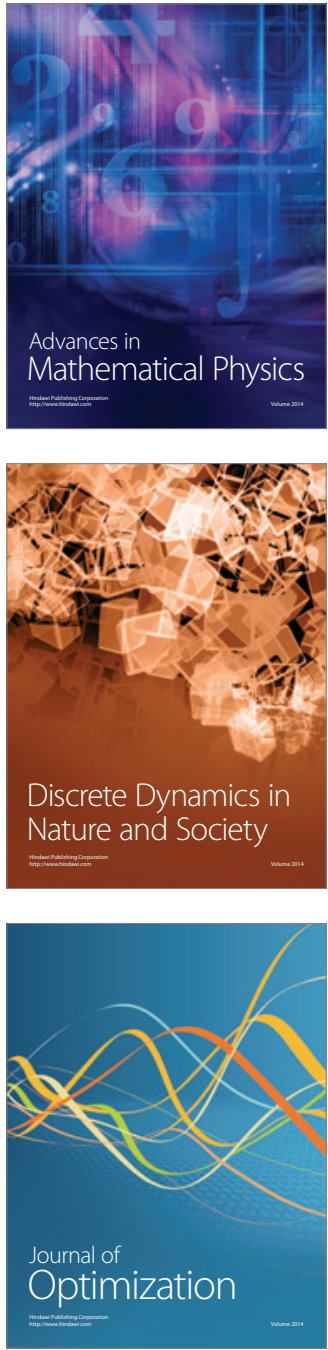